\title{
Machine learning can predict survival of patients with heart failure from serum creatinine and ejection fraction alone
}

\author{
${\text { Davide } \text { Chicco }^{1 *} \text { (D) and Giuseppe Jurman }}^{2}$ (D)
}

\begin{abstract}
Background: Cardiovascular diseases kill approximately 17 million people globally every year, and they mainly exhibit as myocardial infarctions and heart failures. Heart failure (HF) occurs when the heart cannot pump enough blood to meet the needs of the body.

Available electronic medical records of patients quantify symptoms, body features, and clinical laboratory test values, which can be used to perform biostatistics analysis aimed at highlighting patterns and correlations otherwise undetectable by medical doctors. Machine learning, in particular, can predict patients' survival from their data and can individuate the most important features among those included in their medical records.

Methods: In this paper, we analyze a dataset of 299 patients with heart failure collected in 2015 . We apply several machine learning classifiers to both predict the patients survival, and rank the features corresponding to the most important risk factors. We also perform an alternative feature ranking analysis by employing traditional biostatistics tests, and compare these results with those provided by the machine learning algorithms. Since both feature ranking approaches clearly identify serum creatinine and ejection fraction as the two most relevant features, we then build the machine learning survival prediction models on these two factors alone.
\end{abstract}

Results: Our results of these two-feature models show not only that serum creatinine and ejection fraction are sufficient to predict survival of heart failure patients from medical records, but also that using these two features alone can lead to more accurate predictions than using the original dataset features in its entirety. We also carry out an analysis including the follow-up month of each patient: even in this case, serum creatinine and ejection fraction are the most predictive clinical features of the dataset, and are sufficient to predict patients' survival.

Conclusions: This discovery has the potential to impact on clinical practice, becoming a new supporting tool for physicians when predicting if a heart failure patient will survive or not. Indeed, medical doctors aiming at understanding if a patient will survive after heart failure may focus mainly on serum creatinine and ejection fraction.

Keywords: Cardiovascular heart diseases, Heart failure, Serum creatinine, Ejection fraction, Medical records, Feature ranking, Feature selection, Biostatistics, Machine learning, Data mining, Biomedical informatics

\section{Background}

Cardiovascular diseases (CVDs) are disorders of the heart and blood vessels including, coronary heart disease (heart attacks), cerebrovascular diseases (strokes), heart failure (HF), and other types of pathology [1]. Altogether, cardiovascular diseases cause the death of approximately 17

*Correspondence: davidechicco@davidechicco.it

${ }^{1}$ Krembil Research Institute, Toronto, Ontario, Canada

Full list of author information is available at the end of the article million people worldwide annually, with fatalities figures on the rise for first time in 50 years the United Kingdom [2]. In particular, heart failure occurs when the heart is unable to pump enough blood to the body, and it is usually caused by diabetes, high blood pressure, or other heart conditions or diseases [3].

The clinical community groups heart failure into two types based on the ejection fraction value, that is the proportion of blood pumped out of the heart during a single

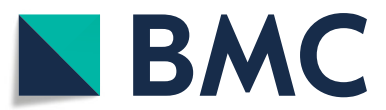

(c) The Author(s). 2020 Open Access This article is distributed under the terms of the Creative Commons Attribution 4.0 International License (http://creativecommons.org/licenses/by/4.0/), which permits unrestricted use, distribution, and reproduction in any medium, provided you give appropriate credit to the original author(s) and the source, provide a link to the Creative Commons license, and indicate if changes were made. The Creative Commons Public Domain Dedication waiver (http://creativecommons.org/publicdomain/zero/1.0/) applies to the data made available in this article, unless otherwise stated. 
contraction, given as a percentage with physiological values ranging between $50 \%$ and $75 \%$. The former is heart failure due to reduced ejection fraction (HFrEF), previously known as heart failure due to left ventricular (LV) systolic dysfunction or systolic heart failure and characterized by an ejection fraction smaller than 40\% [4]. The latter is heart failure with preserved ejection fraction (HFpEF), formerly called diastolic heart failure or heart failure with normal ejection fraction. In this case, the left ventricle contracts normally during systole, but the ventricle is stiff and fails to relax normally during diastole, thus impairing filling [5-10].

For the quantitative evaluation of the disease progression, clinicians rely on the New York Heart Association (NYHA) functional classification, including four classes ranging from no symptoms from ordinary activities (Class I) to a stage where any physical activity brings on discomfort and symptoms occur at rest (Class IV). Despite its widespread use, there is no consistent method of assessing the NYHA score, and this classification fails to reliably predict basic features, such as walking distance or exercise tolerance on formal testing [11].

Given the importance of a vital organ such as the heart, predicting heart failure has become a priority for medical doctors and physicians, but to date forecasting heart failure-related events in clinical practice usually has failed to reach high accuracy [12].

In this context, electronic health records (EHRs, also called medical records) can be considered a useful resource of information to unveil hidden and non-obvious correlations and relationships between patients' data, not only for research but also for clinical practice $[13,14]$ and for debunking traditional myths on risk factors $[15,16]$. To this aim, several screening studies have been conducted in the last years, covering different conditions and demographics and with different data sources, to deepen the knowledge on the risk factors. Among them, it is worth mentioning the PLIC study [17], where EHRs, blood test, single-nucleotide polymorphisms (SNPs), carotid ultrasound imaging, and metagenomics data have been collected in a four-visit longitudinal screening throughout 15 years in Milan (Italy, EU) to support a better assessment of cardiovascular disease risk.

Machine learning applied to medical records, in particular, can be an effective tool both to predict the survival of each patient having heart failure symptoms [18, 19], and to detect the most important clinical features (or risk factors) that may lead to heart failure [20, 21]. Scientists can take advantage of machine learning not only for clinical prediction [22, 23], but also for feature ranking [24]. Computational intelligence, especially, shows its predictive power when applied to medical records $[25,26]$, or coupled with imaging [27-29]. Further, deep learning and meta-analysis studies applied to this field have also recently appeared in the literature [30-33], improving on human specialists' performance [34], albeit showing lower accuracy $(0.75$ versus 0.59$)$.

Modeling survival for heart failure (and CVDs in general) is still a problem nowadays, both in terms of achieving high prediction accuracy and identifying the driving factors. Most of the models developed for this purpose reach only modest accuracy [35], with limited interpretability from the predicting variables [36]. More recent models show improvements, especially if the survival outcome is coupled with additional targets (for example, hospitalization [37]). Although scientists have identified a broad set of predictors and indicators, there is no shared consensus on their relative impact on survival prediction [38]. As pointed out by Sakamoto and colleagues [39], this situation is largely due to a lack of reproducibility, which prevents drawing definitive conclusions about the importance of the detected factors. Further, this lack of reproducibility strongly affects model performances: generalization to external validation datasets is often inconsistent and achieves only modest discrimination. Consequently, risk scores distilled from the models suffer similar problems, limiting their reliability [40]. Such uncertainty has led to the proliferation of new risk scores appearing in the literature in the last years, with mixed results [41-47]. As a partial solution to improve models' effectiveness, recent published studies included cohorts restricted to specific classes of patients (for example, elderly or diabetic) $[48,49]$. These attempts have led to tailored models and risk scores $[50,51]$ with better but still not optimal performance.

In this paper, we analyze a dataset of medical records of patients having heart failure released by Ahmad and colleagues [52] in July 2017. Ahmad and colleagues [52] employed traditional biostatistics time-dependent models (such as Cox regression [53] and Kaplan-Meier survival plots [54]) to predict mortality and identify the key features of 299 Pakistan patients having heart failure, from their medical records. Together with their analysis description and results, Ahmad and coworkers made their dataset publicly available online ("Dataset" section), making it freely accessible to the scientific community [55]. Afterwards, Zahid and colleagues [56] analyzed the same dataset to elaborate two different sex-based mortality prediction models: one for men and one for women. Although the two aforementioned studies [52, 56] presented interesting results, they tackled the problem by standard biostatistics methods, leaving room for machine learning approaches. We aim here to fill this gap by using several data mining techniques first to predict survival of the patients, and then to rank the most important features included in the medical records. As major result, we show that the top predictive performances can be reached by machine learning methods with just two features, none 
of them coming unexpected: one is ejection fraction, and the other is serum creatinine, well known in the literature as a major driver of heart failure [57-62], and also a key biomarker in renal dysfunction [63-65].

In particular, we first describe the analyzed dataset and its features ("Dataset" section), and then the methods we employed for survival prediction and feature ranking ("Methods" section). In the Results section ("Results" section), we report the survival prediction performances obtained through all the employed classifiers ("Survival machine learning prediction on all clinical features" section), the ranking of the features obtained through traditional biostatistics techniques and machine learning ("Feature ranking results" section), and the survival prediction performances achieved by employing only the top two features identified through feature ranking (ejection fraction and serum creatinine, "Survival machine learning prediction on serum creatinine and ejection fraction alone" section). Later, we report and describe the results of the analysis that includes the patients' follow-up time (Table 11). Finally, we discuss the results ("Discussion" section) and draw some conclusions at the end of the manuscript ("Conclusions" section).

\section{Dataset}

We analyzed a dataset containing the medical records of 299 heart failure patients collected at the Faisalabad Institute of Cardiology and at the Allied Hospital in Faisalabad (Punjab, Pakistan), during April-December 2015 [52, 66]. The patients consisted of 105 women and 194 men, and their ages range between 40 and 95 years old (Table 1). All
299 patients had left ventricular systolic dysfunction and had previous heart failures that put them in classes III or IV of New York Heart Association (NYHA) classification of the stages of heart failure [67].

The dataset contains 13 features, which report clinical, body, and lifestyle information (Table 1), that we briefly describe here. Some features are binary: anaemia, high blood pressure, diabetes, sex, and smoking (Table 1). The hospital physician considered a patient having anaemia if haematocrit levels were lower than 36\% [52]. Unfortunately, the original dataset manuscript provides no definition of high blood pressure [52].

Regarding the features, the creatinine phosphokinase (CPK) states the level of the CPK enzyme in blood. When a muscle tissue gets damaged, CPK flows into the blood. Therefore, high levels of CPK in the blood of a patient might indicate a heart failure or injury [68]. The ejection fraction states the percentage of how much blood the left ventricle pumps out with each contraction. The serum creatinine is a waste product generated by creatine, when a muscle breaks down. Especially, doctors focus on serum creatinine in blood to check kidney function. If a patient has high levels of serum creatinine, it may indicate renal dysfunction [69]. Sodium is a mineral that serves for the correct functioning of muscles and nerves. The serum sodium test is a routine blood exam that indicates if a patient has normal levels of sodium in the blood. An abnormally low level of sodium in the blood might be caused by heart failure [70]. The death event feature, that we use as the target in our binary classification study, states if the patient died or survived before the

Table 1 Meanings, measurement units, and intervals of each feature of the dataset

\begin{tabular}{|c|c|c|c|}
\hline Feature & Explanation & Measurement & Range \\
\hline Age & Age of the patient & Years & {$[40, \ldots, 95]$} \\
\hline Anaemia & Decrease of red blood cells or hemoglobin & Boolean & 0,1 \\
\hline High blood pressure & If a patient has hypertension & Boolean & 0,1 \\
\hline Creatinine phosphokinase & Level of the CPK enzyme in the blood & $\mathrm{mcg} / \mathrm{L}$ & {$[23, \ldots, 7861]$} \\
\hline \multicolumn{4}{|l|}{$(\mathrm{CPK})$} \\
\hline Diabetes & If the patient has diabetes & Boolean & 0,1 \\
\hline \multirow[t]{2}{*}{ Ejection fraction } & Percentage of blood leaving & Percentage & {$[14, \ldots, 80]$} \\
\hline & the heart at each contraction & & \\
\hline Sex & Woman or man & Binary & 0,1 \\
\hline Platelets & Platelets in the blood & kiloplatelets/mL & {$[25.01, \ldots .850 .00]$} \\
\hline Serum creatinine & Level of creatinine in the blood & $\mathrm{mg} / \mathrm{dL}$ & {$[0.50, \ldots, 9.40]$} \\
\hline Serum sodium & Level of sodium in the blood & $\mathrm{mEq} / \mathrm{L}$ & {$[114, \ldots, 148]$} \\
\hline Smoking & If the patient smokes & Boolean & 0,1 \\
\hline Time & Follow-up period & Days & {$[4, \ldots, 285]$} \\
\hline (target) death event & If the patient died during the follow-up period & Boolean & 0,1 \\
\hline
\end{tabular}

mcg/L: micrograms per liter. mL: microliter. mEq/L: milliequivalents per litre 
Table 2 Statistical quantitative description of the category features

\begin{tabular}{|c|c|c|c|c|c|c|}
\hline \multirow[b]{2}{*}{ Category feature } & \multicolumn{2}{|c|}{ Full sample } & \multicolumn{2}{|c|}{ Dead patients } & \multicolumn{2}{|c|}{ Survived patients } \\
\hline & $\#$ & $\%$ & $\#$ & $\%$ & $\#$ & $\%$ \\
\hline Anaemia (0: false) & 170 & 56.86 & 50 & 52.08 & 120 & 59.11 \\
\hline Anaemia (1: true) & 129 & 43.14 & 46 & 47.92 & 3 & 40.89 \\
\hline High blood pressure (0: false) & 194 & 64.88 & 57 & 59.38 & 137 & 67.49 \\
\hline High blood pressure (1: true) & 105 & 35.12 & 39 & 40.62 & 66 & 32.51 \\
\hline Diabetes (0: false) & 174 & 58.19 & 56 & 58.33 & 118 & 58.13 \\
\hline Diabetes (1: true) & 125 & 41.81 & 40 & 41.67 & 85 & 41.87 \\
\hline Sex (0: woman) & 105 & 35.12 & 34 & 35.42 & 71 & 34.98 \\
\hline Sex (1: man) & 194 & 64.88 & 62 & 64.58 & 132 & 65.02 \\
\hline Smoking (0: false) & 203 & 67.89 & 66 & 68.75 & 137 & 67.49 \\
\hline Smoking (1: true) & 96 & 32.11 & 30 & 31.25 & 66 & 32.51 \\
\hline
\end{tabular}

\#: number of patients. \%: percentage of patients. Full sample: 299 individuals. Dead patients: 96 individuals. Survived patients: 203 individuals.

end of the follow-up period, that was 130 days on average [52]. The original dataset article [52] unfortunately does not indicate if any patient had primary kidney disease, and provides no additional information about what type of follow-up was carried out. Regarding the dataset imbalance, the survived patients (death event $=0$ ) are 203 , while the dead patients (death event $=1$ ) are 96. In statistical terms, there are $32.11 \%$ positives and $67.89 \%$ negatives.

As done by the original data curators [52], we represented this dataset as a table having 299 rows (patients) and 13 columns (features). For clarification purposes, we slightly changed the names of some features of the original dataset (Additional file 1). We report the quantitative characteristics of the dataset in Table 2 and Table 3. Additional information about this dataset can be found in the original dataset curators publication $[52,66]$.

\section{Methods}

In this section, we first list the machine learning methods we used for the binary classification of the survival ("Survival prediction classifiers" section), and the biostatistics and machine learning methods we employed for the feature ranking ("Feature ranking" section), discarding each patient's follow-up time. We then describe the logistic regression algorithm we employed to predict survival and to perform the feature ranking as a function of the follow-up time ("Stratified logistic regression" section). We implemented all the methods with the open source $\mathrm{R}$ programming language, and made it publically freely available online (Data and software availability).

\section{Survival prediction classifiers}

This part of our analysis focuses on the binary prediction of the survival of the patients in the follow-up period.

To predict patients survival, we employed ten different methods from different machine learning areas. The classifiers include one linear statistical method (Linear Regression [71]), three tree-based methods (Random Forests [72], One Rule [73], Decision Tree [74]), one Artificial Neural Network (perceptron [75]), two Support Vector Machines (linear, and with Gaussian radial kernel [76]), one instance-based learning model ( $k$-Nearest Neighbors [77]), one probabilistic classifier (Naïve Bayes

Table 3 Statistical quantitative description of the numeric features

\begin{tabular}{|c|c|c|c|c|c|c|c|c|c|}
\hline \multirow[b]{2}{*}{ Numeric feature } & \multicolumn{3}{|c|}{ Full sample } & \multicolumn{3}{|c|}{ Dead patients } & \multicolumn{3}{|c|}{ Survived patients } \\
\hline & Median & Mean & $\sigma$ & Median & Mean & $\sigma$ & Median & Mean & $\sigma$ \\
\hline Age & 60.00 & 60.83 & 11.89 & 65.00 & 65.22 & 13.21 & 60.00 & 58.76 & 10.64 \\
\hline Creatinine phosphokinase & 250.00 & 581.80 & 970.29 & 259.00 & 670.20 & 1316.58 & 245.00 & 540.10 & 753.80 \\
\hline Ejection fraction & 38.00 & 38.08 & 11.83 & 30.00 & 33.47 & 12.53 & 38.00 & 40.27 & 10.86 \\
\hline Platelets & 262.00 & 263.36 & 97.80 & 258.50 & 256.38 & 98.53 & 263.00 & 266.66 & 97.53 \\
\hline Serum creatinine & 1.10 & 1.39 & 1.03 & 1.30 & 1.84 & 1.47 & 1.00 & 1.19 & 0.65 \\
\hline Serum sodium & 137.00 & 136.60 & 4.41 & 135.50 & 135.40 & 5.00 & 137.00 & 137.20 & 3.98 \\
\hline Time & 115.00 & 130.30 & 77.61 & 44.50 & 70.89 & 62.38 & 172.00 & 158.30 & 67.74 \\
\hline
\end{tabular}

Full sample: 299 individuals. Dead patients: 96 individuals. Survived patients: 203 individuals. $\sigma$ : standard deviation 
[78]), and an ensemble boosting method (Gradient Boosting [79]).

We measured the prediction results through common confusion matrix rates such as Matthews correlation coefficient (MCC) [80], receiver operating characteristic (ROC) area under the curve, and precision-recall (PR) area under the curve (Additional file 1) [81]. The MCC takes into account the dataset imbalance and generates a high score only if the predictor performed well both on the majority of negative data instances and on the majority of positive data instances [82-84]. Therefore, we give more importance to the MCC than to the other confusion matrix metrics, and rank the results based on the MCC.

\section{Feature ranking}

For the feature ranking, we employed a traditional univariate biostatistics analysis followed by a machine learning analysis; afterwards, we compared the results of the two approaches.

Biostatistics. We used common univariate tests such as Mann-Whitney $U$ test [85], Pearson correlation coefficient [86], and chi square test [87] to compare the distribution of each feature between the two groups (survived individuals and dead patients), plus the Shapiro-Wilk test [88] to check the distribution of each feature. Each test has a different meaning but all of them produce a score (a coefficient for the PCC, and a $p$-value for the other tests) representing the likelihood of a feature to be associated to the target. These scores can then be employed to produce a ranking, that lists the features from the most target-related to the least target-related.

The Mann-Whitney $U$ test (or Wilcoxon rank-sum test) [85], applied to each feature in relation to the death event target, detects whether we can reject the null hypothesis that the distribution of the each feature for the groups of samples defined by death event are the same. A low $p$-value of this test (close to 0 ) means that the analyzed feature strongly relates to death event, while a high $p$-value (close to 1) means the opposite. The Pearson correlation coefficient (or Pearson product-moment correlation coefficient, PCC) [86] indicates the linear correlation between elements of two lists, showing the same elements on different positions. The absolute value of PCC generates a high value (close to 1 ) if the elements of the two lists have linear correlation, and a low value (close to 0 ) otherwise.

The chi square test (or $\chi^{2}$ test) [87] between two features checks how likely an observed distribution is due to chance [89]. A low $p$-value (close to 0 ) means that the two features have a strong relation; a high $p$-value (close to 1 ) means, instead, that the null hypothesis of independence cannot be discarded.

Similar to what Miguel and colleagues did on a breast cancer dataset [90], we decided also to take advantage of the Shapiro-Wilk test [88] to assess if each feature was extracted from a normal distribution.

Machine learning. Regarding machine learning feature ranking, we focused only on Random Forests [72, 91], because as it turned out to be the top performing classifier on the complete dataset ("Feature ranking results" section). Random Forests [72] provides two feature ranking techniques: mean accuracy reduction and Gini impurity reduction [92]. During training, Random Forests generates several random Decision Trees that it applies to data subsets, containing a subsets both of data instances and of features. In the end, Random Forests checks all the binary outcomes of these decisions trees and chooses its final outcome through a majority vote. The feature ranking based upon the mean accuracy decreases counts how much the prediction accuracy decreases, when a particular feature is removed. The method then compares this accuracy with the accuracy obtained by using all the features, and considers this difference as the importance of that specific feature: the larger the accuracy drop, the more important the feature. The other feature ranking method works similarly, but is based upon the Gini impurity decrease [91]: the more the Gini impurity drops, the more important the feature.

\section{Aggregate feature rankings and prediction on the top features}

Starting from the whole dataset $D$ we generated a collection $\mathcal{D}=\left\{\left\{D_{i}^{\mathrm{tr}}, D_{i}^{\mathrm{ts}}\right\}\right\}_{i=1}^{N}$ of $N$ Monte Carlo stratified training/test partitions $D=D_{i}^{\text {tr }} \cup D_{i}^{\text {ts }}$ with ratio $70 \% / 30 \%$.

For each execution, we randomly selected $70 \%$ of patients for the training set, and used the remaining 30\% for the test set. To make our predictions more realistic, we avoided using the same balance ratio of the whole complete dataset (32.11\% positives and $67.89 \%$ negatives). This way, we had different balance ratios for each of the 100 executions with, on average, $32.06 \%$ positives and $66.94 \%$ negatives on average in the training sets, and with, on average, $32.22 \%$ positives and $67.78 \%$ negatives on average in the test sets.

On the $N$ training portions $D_{1}^{\mathrm{tr}}, \ldots, D_{N}^{\mathrm{tr}}$ we applied seven different feature ranking methods, namely RReliefF [93-95], Max-Min Parents and Children [96-98], Random Forest [72], One Rule [73], Recursive Partitioning and Regression Trees [99], Support Vector Machines with linear kernel [100] and eXtreme Gradient Boosting $[79,101,102]$, using the feature death event as the target and obtaining $7 N$ ranked lists of the 11 features. Agglomerating all the $7 \mathrm{~N}$ features into the single Borda list [103, 104] we obtained the global list (Fig. 2 for $N=$ $100)$, together with the Borda count score of each feature, corresponding to the average position across all $7 \mathrm{~N}$ lists, and thus the lower the score, the more important the feature. 
We then used only the top-two features, namely serum creatinine and ejection fraction to build on each subset $D_{i}^{\text {tr }}$ three classifiers, namely Random Forests (RF), Support Vector Machine with Gaussian Kernel (GSVM) and eXtreme Gradient Boosting (XGB). Finally, we then applied the trained models to the corresponding test portions $D_{i}^{\text {ts }}$ with the aforementioned top-2 features and averaged the obtained performances modelwise on the $N$ test set instances.

For the feature ranking and the classification made on the top two features, we employed different sets of the machine learning methods than the ones we used for the survival prediction on the complete dataset ("Survival prediction classifiers" section): RReliefF, MaxMin Parents and Children, Random Forests, One Rule, Recursive Partitioning and Regression Trees Support Vector Machines with linear kernel, and eXtreme Gradient Boosting, for the feature ranking, and Random Forests, Gradient Boosting, and SVM with radial kernel. We decided to use three different sets of methods because we aimed to demonstrate the generalisability of our approach, by showing that our computational solution is not only valid with few machine learning classifiers, but rather works for several groups of methods.

Regarding the final prediction using only the top two selected features, we chose Random Forests because it resulted in being the top performing classifier on the complete feature dataset ("Survival machine learning prediction on all clinical features" section) and it is universally considered an efficient method for feature ranking [92]. We then chose Gradient Boosting and Support Vector Machine with radial Gaussian kernel because both these methods have shown efficient performances in feature ranking with medical informatics data $[105,106]$.

\section{Stratified logistic regression}

In the just-described first analysis, we wanted to predict the survival of patients and to detect the clinical feature importance in the follow-up time, without considering its different extent for each patient. In the second analysis, we decided to include the follow-up time, to see if the survival prediction results or the feature ranking results would change. To analyze this aspect, we mapped the original dataset time feature (containing the days of follow-up) into a month variable, where month 0 means that fewer than 30 days have gone by, month 1 means between 30 and 60 days, month 2 means between 60 and 90 days, and so on.

We then applied a stratified logistic regression [107] to the complete dataset, including all the original clinical features and the derived follow-up month feature. We measured the prediction with the aforementioned confusion matrix metrics ( $\mathrm{MCC}, \mathrm{F}_{1}$ score, etc.), and the feature ranking importance as the logistic regression model coefficient for each variable.

\section{Results}

In this section, we first describe the results we obtained for the survival prediction on the complete dataset ("Survival machine learning prediction on all clinical features" section), the results obtained for the feature ranking ("Feature ranking results" section), and the results on the survival prediction when using only the top two most important features of the dataset ("Survival machine learning prediction on serum creatinine and ejection fraction alone" section and "Serum creatinine and ejection fraction linear separability" section), all independently from the follow-up time. We then report and discuss the results achieved by including the follow-up time of each patient in the survival prediction and feature ranking ("Survival prediction and feature ranking including the follow-up period" section).

Survival machine learning prediction on all clinical features We employed several methods to predict the survival of the patients. We applied each method 100 times and reported the mean result score (Table 4).

For methods that needed hyper-parameter optimization (neural network, Support Vector Machine, and $k$-Nearest Neighbors), we split the dataset into 60\% (179 randomly selected patients) for the training set, 20\% (60 randomly selected patients) for the validation set, and $20 \%$ (the remaining 60 patients) for the test set. To choose the top hyper-parameters, we used a grid search and selected the models that generated the highest Matthews correlation coefficient [83].

For the other methods (Random Forests, One Rule, Linear Regression, Naïve Bayes, and Decision Tree), instead, we split the dataset into $80 \%$ (239 randomly selected patients) for the training set, and $20 \%$ (the remaining 60 patients) for the test set.

For each of the 100 executions, our script randomly selected data instances for the training set and for the test (and for the validation set, in the case of hyper-parameter optimization) from the complete original dataset. We trained the model on the training set (and validated it on the validation set, in the case of hyper-parameter optimization). We then applied the script to the test set. Given the different selections of data instances for the dataset splits, each execution led to slightly different results.

Our prediction results showed that Random Forests outperformed all the other methods, by obtaining the top MCC (+0.384), the top accuracy (0.740), and the top ROC AUC (0.800) (Table 4). The Decision Trees obtained the top results on the true positives (sensitivity $=0.532$ ) and on the $\mathrm{F}_{1}$ score $(0.554)$, and was the only classifier able to predict correctly the majority of deceased patients. The 
Table 4 Survival prediction results on all clinical features - mean of 100 executions

\begin{tabular}{|c|c|c|c|c|c|c|c|}
\hline Method & MCC & $F_{1}$ score & Accuracy & TP rate & $\mathrm{TN}$ rate & PR AUC & ROC AUC \\
\hline Random forests & $+0.384^{*}$ & 0.547 & $0.740^{*}$ & 0.491 & 0.864 & 0.657 & $0.800^{*}$ \\
\hline Decision tree & +0.376 & $0.554^{*}$ & 0.737 & $0.532^{*}$ & 0.831 & 0.506 & 0.681 \\
\hline Gradient boosting & +0.367 & 0.527 & 0.738 & 0.477 & 0.860 & 0.594 & 0.754 \\
\hline Linear regression & +0.332 & 0.475 & 0.730 & 0.394 & 0.892 & 0.495 & 0.643 \\
\hline One rule & +0.319 & 0.465 & 0.729 & 0.383 & 0.892 & 0.482 & 0.637 \\
\hline Artificial neural network & +0.262 & 0.483 & 0.680 & 0.428 & 0.815 & $0.750^{*}$ & 0.559 \\
\hline Naïve bayes & +0.224 & 0.364 & 0.696 & 0.279 & 0.898 & 0.437 & 0.589 \\
\hline SVM radial & +0.159 & 0.182 & 0.690 & 0.122 & 0.967 & 0.587 & 0.749 \\
\hline SVM linear & +0.107 & 0.115 & 0.684 & 0.072 & $0.981^{*}$ & 0.594 & 0.754 \\
\hline k-nearest neighbors & -0.025 & 0.148 & 0.624 & 0.121 & 0.866 & 0.323 & 0.493 \\
\hline
\end{tabular}

MCC: Matthews correlation coefficient. TP rate: true positive rate (sensitivity, recall). TN rate: true negative rate (specificify). Confusion matrix threshold for MCC, $\mathrm{F}_{1} \mathrm{score}$, accuracy, TP rate, TN rate: $\tau=0.5$. PR: precision-recall curve. ROC: receiver operating characteristic curve. AUC: area under the curve. MCC: worst value $=-1$ and best value $=$ +1. F1 score, accuracy, TP rate, TN rate, PR AUC, ROC AUC: worst value $=0$ and best value $=1$. MCC, F1 score, accuracy, TP rate, TN rate, PR AUC, ROC AUC formulas: Additional file 1 ("Binary statistical rates" section). Gradient boosting: eXtreme Gradient Boosting (XGBoost). SVM radial: Support Vector Machine with radial Gaussian kernel. SVM linear: Support Vector Machine with linear kernel. Our hyper-parameter grid search optimization for $k$-Nearest Neighbors selected $k=3$ on most of the times (10 runs out of 100). Our hyper-parameter grid search optimization for the Support Vector Machine with radial Gaussian kernel selected C $=10$ on most of the times (56 runs out of 100). Our hyper-parameter grid search optimization for the Support Vector Machine with linear kernel selected $C=0.1$ on most of the times (50 runs out of 100 ). Our hyper-parameter grid search optimization for the Artificial Neural Network selected 1 hidden layer and 100 hidden units on most of the times (74 runs out of 100 ). We report in blue and with * the top performer results for each score.

linear Support Vector Machines achieved an almost perfect prediction score on the negative elements (specificity $=0.961$ ), but a poor score on the positive elements (sensitivity $=0.072$ ). The Artificial Neural Network perceptron, instead, obtained the top value on the Precision-Recall AUC (0.750).

Because of the imbalance of the dataset $(67.89 \%$ negative elements and $32.11 \%$ positive elements), all the methods obtained better prediction scores on the true negative rate, rather than on the true positive rate (Table 4). These results occur because the algorithms can see more negative elements during training, and therefore they are more trained to recognize deceased patient profiles during testing.

\section{Feature ranking results}

Similarly to what authors did for a dataset of patients having mesothelioma symptoms [92], we decided then to investigate the most important features of the cardiovascular heart disease patients dataset. To this aim, we first performed a traditional univariate biostatistics analysis ("Feature ranking" section), and then employed Random Forests [108], to generate machine learning results. We then compared the results obtained through the two approaches.

All the biostatistics tests (Mann-Whitney $U$ test in Table 5, Pearson correlation coefficient in Table 6, and chi squared test in Table 7) identified serum creatinine and ejection fraction as the top two most important features.

Mann-Whitney $U$ test (Table 5) and chi squared test (Table 7), in particular, showed a significant $p$-value close to 0 for both these two features. The Pearson correlation coefficient results (Table 6, left side) also showed age, in the third position, as a top feature among serum creatinine and ejection fraction.

The Shapiro-Wilk test (Table 6, right side) generated $p$ values close to 0 for all the features, meaning that the null hypothesis of normality is rejected, and all variables are non-normal.

Regarding Random Forests feature ranking, both the accuracy reduction and the Gini impurity rankings detected serum creatinine, ejection fraction, and age as the top three most important features of the dataset (Fig. 1). The two rankings show high similarity: the

Table 5 Mann-Whitney $U$ test

\begin{tabular}{lll}
\hline Rank & Feature & $\begin{array}{l}\text { Mann-Whitney } U \\
\text { Test } p \text {-value }\end{array}$ \\
\hline 1 & Serum creatinine & 0 \\
2 & Ejection fraction & 0.000001 \\
3 & Age & 0.000167 \\
4 & Serum sodium & 0.000293 \\
5 & High blood pressure & 0.171016 \\
6 & Anaemia & 0.252970 \\
7 & Platelets & 0.425559 \\
8 & Creatinine phosphokinase & 0.684040 \\
9 & Smoking & 0.828190 \\
10 & Sex & 0.941292 \\
11 & Diabetes & 0.973913 \\
\hline
\end{tabular}

Results of the univariate application of the Mann-Whitney $U$ test between each feature and the target feature death event 
Table 6 Pearson correlation coefficients (PCC) and Shapiro-Wilk tests

\begin{tabular}{llllll}
\hline \multicolumn{2}{c}{ Pearson correlation coefficient } & & \multicolumn{2}{l}{ Shapiro-Wilk test } \\
Rank & Feature & abs(PCC) & Rank & Feature & $p$-value \\
\hline 1 & Serum creatinine & 0.294 & 1 & Creatinine phosphokinase & $7.05 \times 10^{-28}$ \\
2 & Ejection fraction & 0.269 & 2 & Serum creatinine & $5.39 \times 10^{-27}$ \\
3 & Age & 0.254 & 3 & Smoking & $4.58 \times 10^{-26}$ \\
4 & 0.195 & 4 & Death event & $4.58 \times 10^{-26}$ \\
5 & Serum sodium & 0.079 & 5 & Sex & $1.17 \times 10^{-25}$ \\
6 & High blood pressure & 0.066 & 6 & High blood pressure & $1.17 \times 10^{-25}$ \\
7 & Anaemia & 0.063 & 7 & Diabetes & $5.12 \times 10^{-25}$ \\
8 & Creatinine phosphokinase & 0.049 & 8 & Anaemia & $6.21 \times 10^{-25}$ \\
9 & Platelets & 0.013 & 9 & Platelets & $2.89 \times 10^{-12}$ \\
10 & Smoking & 0.004 & 10 & Serum sodium & $9.21 \times 10^{-10}$ \\
& Sex & 0.002 & 11 & Ejection fraction & $7.22 \times 10^{-09}$ \\
\hline
\end{tabular}

Results of the univariate application of the Pearson correlation coefficient between each feature and the target feature death event, absolute value (left), and the univariate application of the Shapiro-Wilk test on each feature (right)

Kendall $\tau$ rank correlation coefficient between them is +0.56 and the Spearman $\rho$ rank correlation coefficient is +0.73 . Both these coefficients range between -1 (when the ranking of a list is the opposite of the other one) and +1 (when the two rankings are similar) [109].

To have a unique final classification to evaluate, we then merged the two rankings into an aggregate ranking by using Borda's method [110]. For every feature $f$, we added its position in the accuracy decrease ranking $p_{1}(f)$ to its position in the Gini impurity raking $p_{2}(f)$, and saved this aggregate value in the ranking variable score $_{f}$. Finally, we sorted all the features increasingly based upon score $_{f}$ (Table 8).

Table 7 Chi squared test

\begin{tabular}{lll}
\hline \multicolumn{2}{c}{ Chi squared test } & \\
Rank & Feature & $p$-value \\
\hline 1 & Ejection fraction & 0.000500 \\
2 & Serum creatinine & 0.000500 \\
3 & Serum sodium & 0.003998 \\
4 & Age & 0.005997 \\
5 & High blood pressure & 0.181909 \\
6 & Anaemia & 0.260370 \\
7 & Creatinine phosphokinase & 0.377811 \\
8 & Platelets & 0.637681 \\
9 & Smoking & 0.889555 \\
10 & Sex & 1 \\
11 & Diabetes & 1 \\
\hline
\end{tabular}

Results of the application of the chi squared test between each feature and the target feature death event
In the aggregated ranking (Table 8), creatinine phosphokinase appeared as the fourth most important feature tied with serum sodium, while anaemia and diabetes were the least important features among all.

Once we obtained the ranking of the features based upon their importance, we aimed to understand what is the minimum number of features (and which features should be used) to still be able to perform an accurate prediction of the survival of patients. In fact, we want to provide a method that can be used by medical doctors in the hospital, in the scenario where just few features of the electronic health record (EHR) of a patient are available.

Since we observed that serum creatinine and ejection fraction resulted as the top two features in the univariate biostastitics analysis tests (Pearson correlation coefficient in Table 6, Mann-Whitney $U$ test in Table 5 and chi squared in Table 7), and in the Random Forests analysis (Table 8 and Fig 1), we decided to explore if it is possible to correctly predict the patients' survival from these top two clinical features alone.

\section{Survival machine learning prediction on serum creatinine and ejection fraction alone}

As mentioned earlier ("Aggregate feature rankings and prediction on the top features" section), we decided to investigate if machine learning can precisely predict patients' survival by using the top two ranked features alone. We therefore elaborated another computational pipeline with an initial phase of feature ranking, followed by a binary classification phase based on the top two features selected (Table 9).

All the different methods employed for feature ranking identified serum creatinine and ejection fraction as 

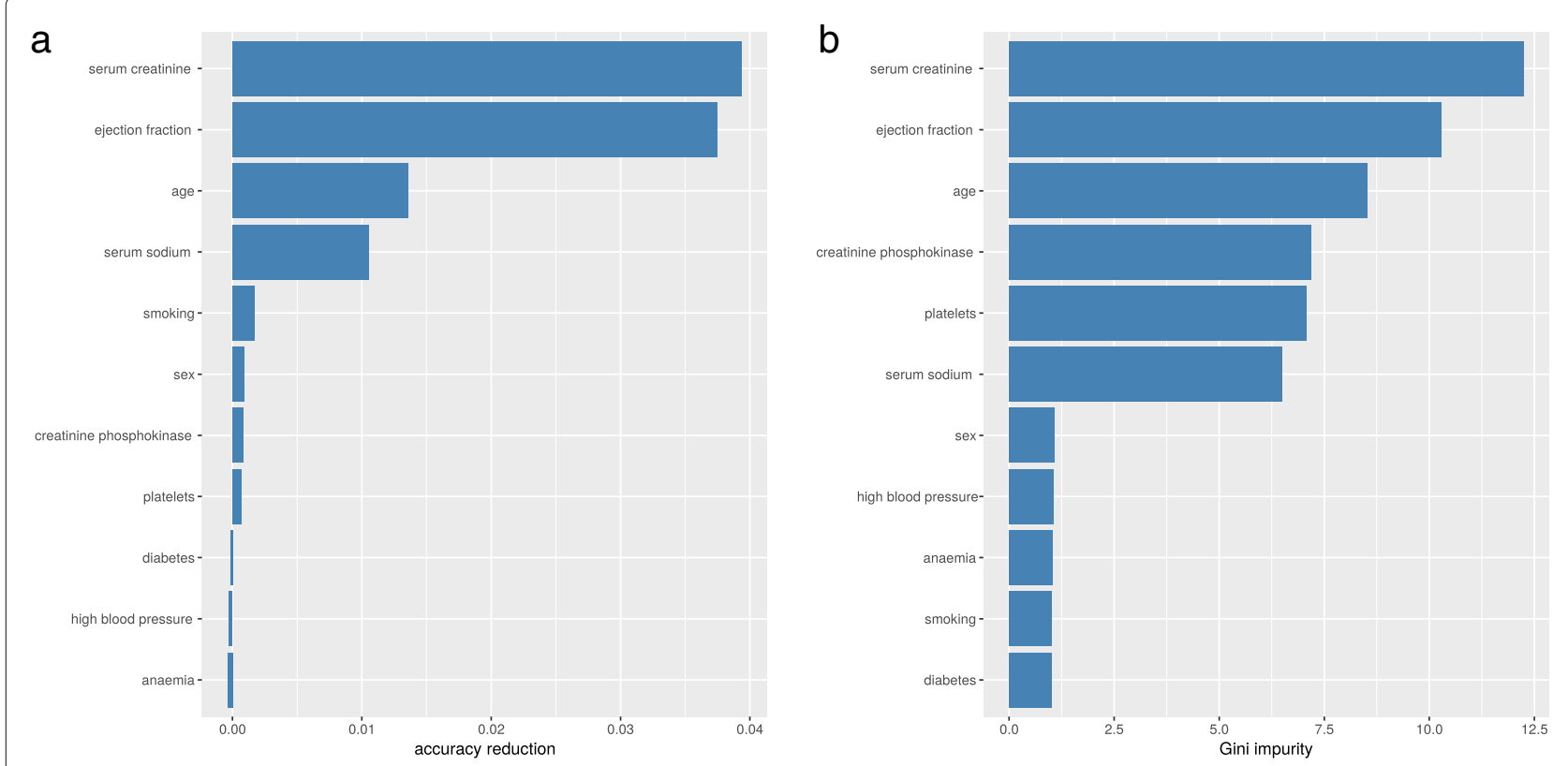

Fig. 1 Random Forests feature selection. Accuracy reduction. Gini impurity. Random Forests feature selection through accuracy reduction (a). Random Forests feature selection through Gini impurity (b)

the top two features for all the 100 executions (Fig. 2), so we then performed a survival prediction on these two features by employing three algorithms: Random Forests, Gradient Boosting, and SVM radial.

All the three classifiers employed outperformed their corresponding results obtained on the complete datase (Table 4). Random Forests and Gradient Boosting applied to serum creatinine and ejection fraction alone, moreover, even outperformed all the methods applied to the complete dataset (Table 4), by achieving Matthews correlation coefficients greater than +0.4 in the $[-1 ;+1]$ range. Similar to the application on the complete dataset, here the classifiers obtained better results on the specificity (true negative rate) than on the recall (true positive rate), because of the imbalance of the dataset.

\section{Serum creatinine and ejection fraction linear separability}

To verify further the predictive power of serum creatinine and ejection fraction, we depicted a scatterplot with the serum creatinine values on the $x$ axis and the ejection fraction values on the $y$ axis, and we colored every patientpoint based on survival status (survived or dead, Fig. 3). This plot shows a clear distinction between alive patients and dead patients, that we highlighted by manually inserting a black straight line.

Table 8 Random Forests feature selection aggregate ranking

\begin{tabular}{|c|c|c|c|c|c|}
\hline Final rank & Feature & Accuracy decrease & Accuracy decrease rank & Gini impurity & Gini impurity rank \\
\hline 1 & Serum creatinine & $3.78 \times 10^{-2}$ & 1 & 11.84 & 1 \\
\hline 2 & Ejection fraction & $3.43 \times 10^{-2}$ & 2 & 10.71 & 2 \\
\hline 3 & Age & $1.53 \times 10^{-2}$ & 3 & 8.58 & 3 \\
\hline 4 & Creatinine phosphokinase & $7.27 \times 10^{-4}$ & 6 & 7.26 & 4 \\
\hline 4 & Serum sodium & $7.20 \times 10^{-3}$ & 4 & 6.49 & 6 \\
\hline 6 & Sex & $1.64 \times 10^{-3}$ & 5 & 1.12 & 8 \\
\hline 6 & Platelets & $2.47 \times 10^{-4}$ & 8 & 6.80 & 5 \\
\hline 8 & High blood pressure & $-1.68 \times 10^{-3}$ & 11 & 1.13 & 7 \\
\hline 8 & Smoking & $3.68 \times 10^{-4}$ & 7 & 0.95 & 11 \\
\hline 10 & Anaemia & $-5.91 \times 10^{-4}$ & 10 & 1.06 & 9 \\
\hline 10 & Diabetes & $-1.41 \times 10^{-4}$ & 9 & 1.02 & 10 \\
\hline
\end{tabular}

We merged the two rankings through their position, through the Borda's method [103] 
Table 9 Survival prediction results on serum creatinine and ejection fraction - mean of 100 executions

\begin{tabular}{llllllll}
\hline Method & MCC & F $_{1}$ score & Accuracy & TP rate & TN rate & PR AUC & ROC AUC \\
\hline Random forests & $\mathbf{+ 0 . 4 1 8 *}$ & $0.754^{*}$ & $0.585^{*}$ & 0.541 & $0.855^{*}$ & 0.541 & 0.698 \\
Gradient boosting & $\mathbf{+ 0 . 4 1 4}$ & 0.750 & $0.585^{*}$ & $0.550^{*}$ & 0.845 & $0.673^{*}$ & $0.792^{*}$ \\
SVM radial & $\mathbf{+ 0 . 3 4 8}$ & 0.720 & 0.543 & 0.519 & 0.816 & 0.494 & 0.667 \\
\hline
\end{tabular}

MCC: Matthews correlation coefficient. TP rate: true positive rate (sensitivity, recall). TN rate: true negative rate (specificify). Confusion matrix threshold for MCC, $\mathrm{F}_{1}$ score, accuracy, TP rate, TN rate: $\tau=0.5$. PR: precision-recall curve. ROC: receiver operating characteristic curve. AUC: area under the curve. MCC: worst value $=-1$ and best value $=$ +1. F 1 score, accuracy, TP rate, TN rate, PR AUC, ROC AUC: worst value $=0$ and best value $=1$. MCC, F 1 score, accuracy, TP rate, TN rate, PR AUC, ROC AUC formulas: Additional file 1 ("Binary statistical rates" section). Gradient boosting: eXtreme Gradient Boosting (XGBoost). SVM radial: Support Vector Machine with radial Gaussian kernel. We reported in blue and with * the top results for each score.

\section{Survival prediction and feature ranking including the follow-up period}

In the previous part of the analysis, we excluded follow-up time from the dataset because we prefered to focus on the clinical features and to try to discover something meaningful about them. Follow-up time, however, can be an important factor in the survival of patients, and should not be eliminated completely from this study. We therefore decided to investigate the possible relationship between follow-up time and the survival of patients: is the moment of the follow-up visit related to the chance of survival of the patient?

Follow-up time and survival. To analyze this aspect, we first grouped together all the surviving patients and the deceased patients for each month. We then built a barplot that relates the percentage of surviving patients to each follow-up month (Fig. 4). This plot shows that it is impossible to correlate the survival of patients to the follow-up month because the survival trend is not linear: the month
5 , in fact, reports less surviving patients than month 4 and month 6 (Fig. 4).

For the same reasons, there is no trend showing an increasing or decreasing rate of survived patients as function of months gone by: the month 5 , in fact, has less half survived patients, similarly to month 0 and month 1 , without being adjacent to them (Fig. 4).

Survival prediction including follow-up time. Even if we notice no linear correlation between follow-up month and survival, we decided to repeat the survival prediction analysis and the feature ranking analysis by including this feature, and to explore the relevance of ejection fraction and serum creatinine in this case. As mentioned earlier ("Stratified logistic regression" section), we used a stratified logistic regression for this task.

We first applied the logistic regression to rank all the clinical features. The results we obtained (Table 10) again showed ejection fraction and serum creatinine to be the most relevant clinical factors.

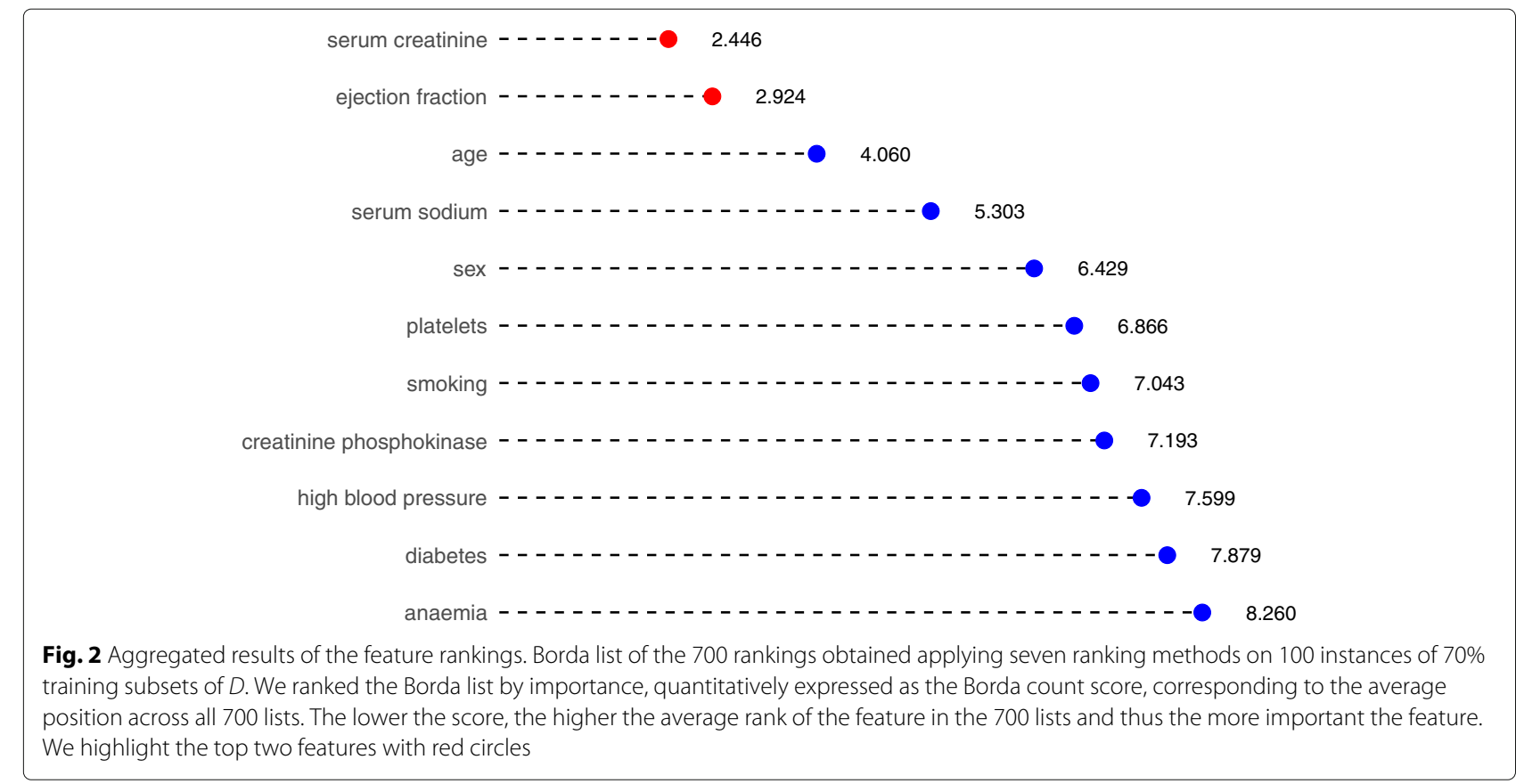




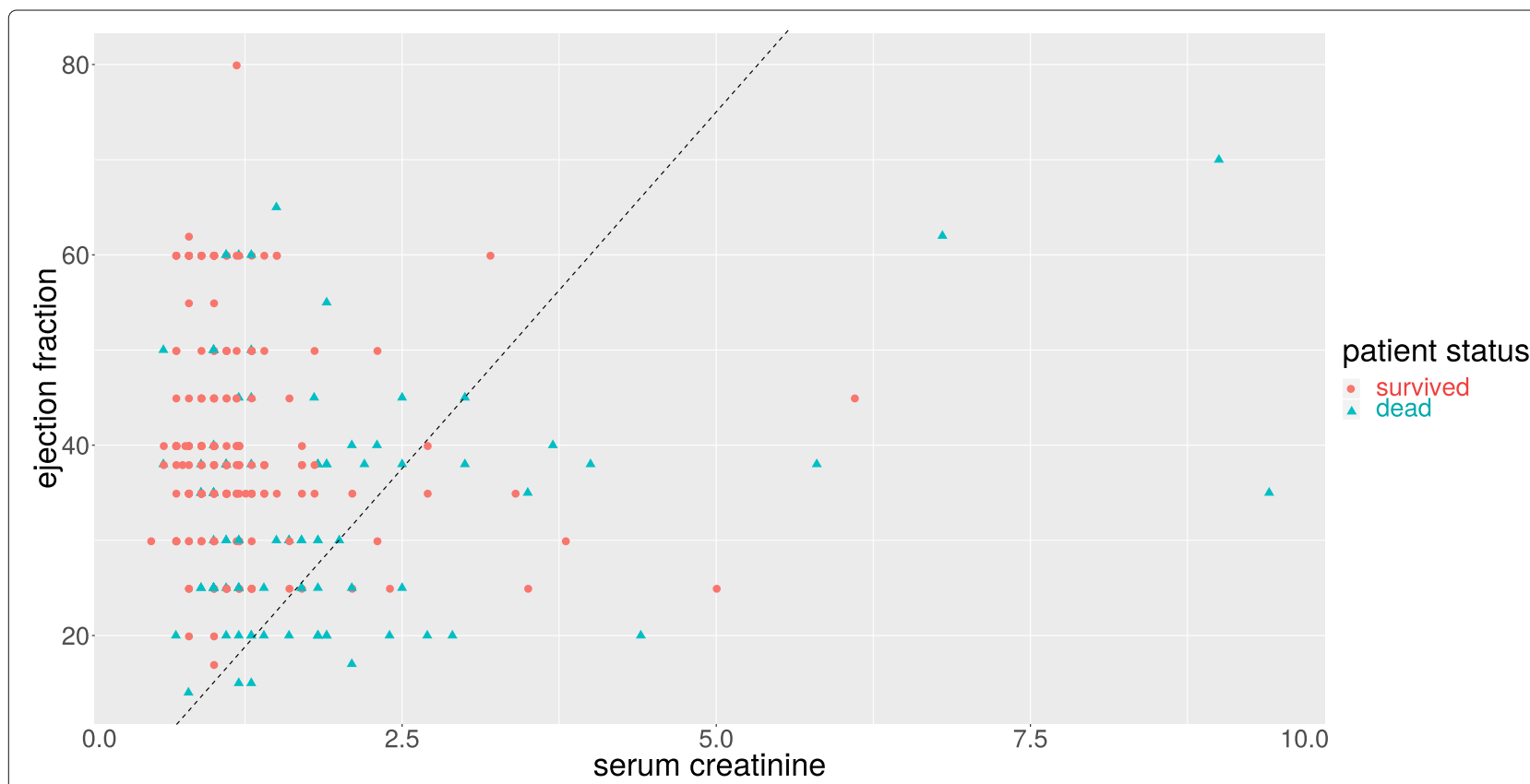

Fig. 3 Scatterplot of serum creatinine versus ejection fraction. Serum creatinine (x axis) range: $[0.50,9.40] \mathrm{mg} / \mathrm{dL}$. Ejection fraction (y axis) range: $[14,80] \%$. We manually drew a black straight line to highlight the discrimination between alive and dead patients

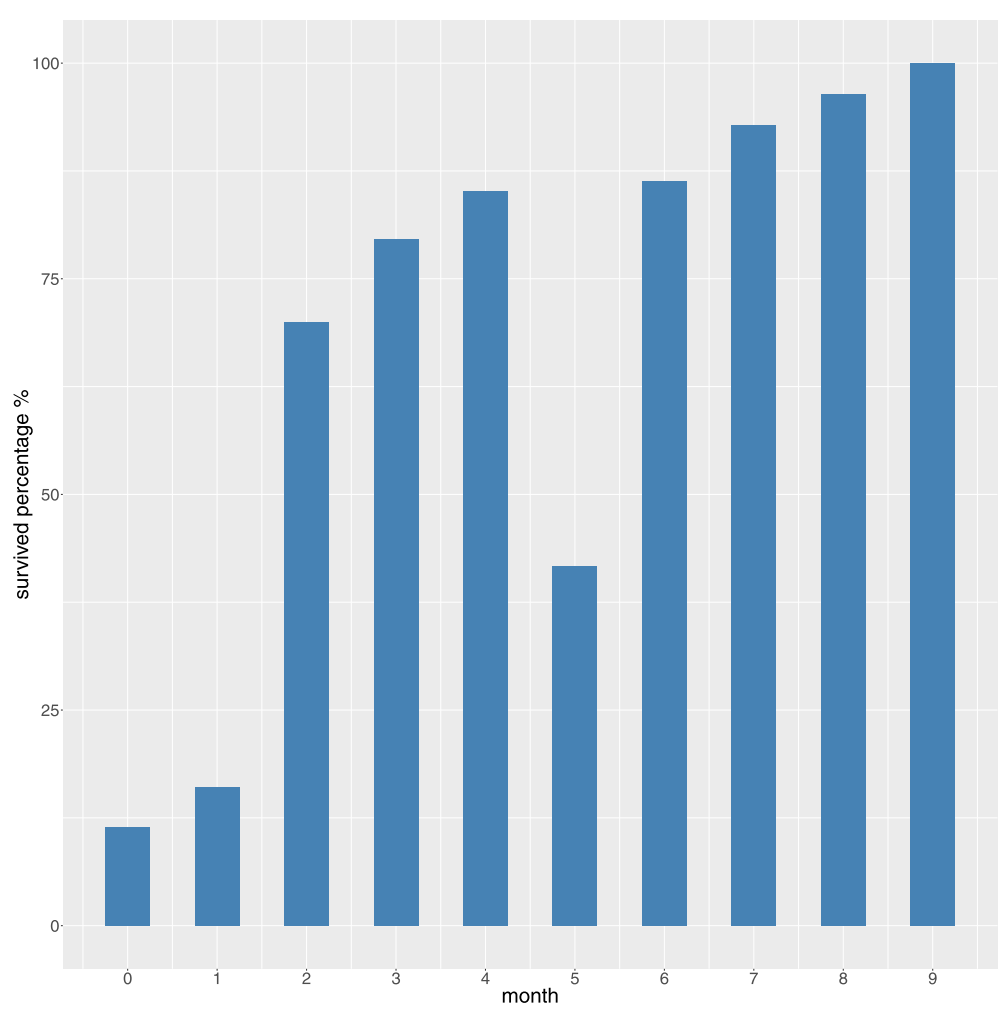

Fig. 4 Barplot of the survival percentage for each follow-up month. Follow-up time (x axis) range: $[0,9]$ months. Survival percentage (y axis) range: $[11.43,100] \%$. For each month, we report here the percentage of survived patients. For the 0 month (less than 30 days), for example, there were $11.43 \%$ survied patients and $88.57 \%$ deceased patients 
Table 10 Stratified logistic regression feature ranking

\begin{tabular}{lll}
\hline Rank & Clinical feature & Importance \\
\hline 1 & Ejection fraction & 4.13938106 \\
2 & Serum creatinine & 3.69917184 \\
3 & Age & 2.61938095 \\
4 & Creatinine phosphokinase & 1.88929235 \\
5 & Sex & 1.32038950 \\
6 & Platelets & 1.06270364 \\
7 & High blood pressure & 0.79478093 \\
8 & Anaemia & 0.77547306 \\
9 & Smoking & 0.65828165 \\
10 & Diabetes & 0.60355319 \\
11 & Serum sodium & 0.54241360 \\
\hline
\end{tabular}

Results of the feature ranking obtained by the stratified logisitc regression. Importance: coefficient of the trained logistic regression model, average of 100 execution

We trained the model on the whole dataset, and then ranked the non-temporal features based upon their generalized linear model (GLM) coefficients. We repeated this operation 100 times and reported the average importance value for each variable (Table 10).

Afterwards, in a similar way as we did before ("Aggregate feature rankings and prediction on the top features" section), we decided to investigate how the survival prediction would behave when using only the two selected features. We trained the stratified logistic regression on all the features including the follow-up time, by using $70 \%$ of patients, randomly selected. We then selected the top two clinical features, trained a model by using only these top two features and follow-up month, and tested this threefeature model on the test set. We applied this method 100 times, and reported the average results (Table 11). For all executions, the top two features were ejection fraction and serum creatinine.

The stratified logistic regression using only three features outperformed the model using all features, in each confusion matrix. metric (Table 10). The results showed that, when including follow-up month into the model, machine learning predictions using only ejection fraction and serum creatinine outperformed a prediction using all the clinical features.

Additionally, the results obtained by the stratified logistic regression and considering the follow-up month (Table 10) outperformed the results achieved by the other methods without the follow-up month (Table 4 and Table 9), highlighting the importance of this temporal variable.

\section{Discussion}

Our results not only show that B it might be possible to predict the survival of patients with heart failure solely from their serum creatinine and ejection fraction, but also that the prediction made on these two features alone can be more accurate than the predictions made on the complete dataset. This aspect is particularly encouraging for the hospital settings: in case many laboratory test results and clinical features were missing from the electronic health record of a patient, doctors could still $\mathrm{B}$ be able to predict patient survival by just analyzing the ejection fraction and serum creatinine values. B That being said, we recognize that additional confirmatory studies need to be completed before this machine learning procedure can be taken up into clinical practice.

Our analysis also generated some interesting results that differ from the original dataset curators study [52]. Ahmad and colleagues, in fact, identified age, serum creatinine (renal dysfunction), high blood pressure, ejection fraction and anaemia as top features. In our Random Forests feature ranking instead (Table 8), high blood pressure is on $8^{\text {th }}$ position out of 11 , and anaemia is on the $10^{\text {th }}$ position out of 11 (last position tied with diabetes).

\section{Conclusions}

In our work, the fact that our traditional biostatistics analysis selected ejection fraction and serum creatinine as the two most relevant features confirmed the relevance of the feature ranking executed with machine learning. Moreover, our approach showed that machine learning can be used effectively for binary classification of electronic health records of patients with cardiovascular hearth diseases.

Table 11 Survival prediction results including the follow-up time - mean of 100 executions

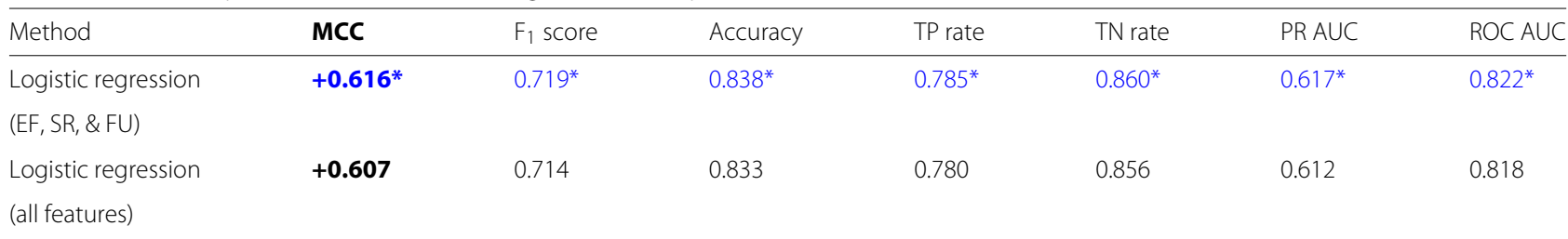

Top row: logistic regression using only ejection fraction (EF), serum creatinine (SC), and follow-up time month (FU). Bottom row: logistic regression using all features. MCC: Matthews correlation coefficient. TP rate: true positive rate (sensitivity, recall). TN rate: true negative rate (specificify). Confusion matrix threshold for MCC, $\mathrm{F}_{1}$ Score, accuracy, TP rate, TN rate: $\tau=0.5$. PR: precision-recall curve. ROC: receiver operating characteristic curve. AUC: area under the curve. MCC: worst value $=-1$ and best value $=+1 . F_{1}$ score, accuracy, TP rate, TN rate, PR AUC, ROC AUC: worst value $=0$ and best value $=1$. MCC, F 1 score, accuracy, TP rate, TN rate, PR AUC, ROC AUC formulas: Additional file 1 ("Binary statistical rates" section). We reported in blue and with * the top results for each score. 
As a limitation of the present study, we have to report the small size of the dataset (299 patients): a larger dataset would have permitted us to obtain more reliable results. Additional information about the physical features of the patients (height, weight, body mass index, etc.) and their occupational history would have been useful to detect additional risk factors for cardiovascular health diseases. Also, if an additional external dataset with the same features from a different geographical region had been available, we would have used it as a validation cohort to verify our findings.

Regarding future developments, we plan to apply our machine learning approach to alternative datasets of cardiovascular heart diseases [111-113] and other illnesses (cervical cancer [114], neuroblastoma [115], breast cancer [90], and amyotrophic lateral sclerosis [116]).

\section{Supplementary information}

Supplementary information accompanies this paper at https://doi.org/10.1186/s12911-020-1023-5.

\section{Additional file 1: Supplementary information.}

\section{Abbreviations}

AUC: area under the curve; CPK: creatinine phosphokinase; CVDs: cardiovascular diseases; EF: ejection fraction; EHR: electronic health records; EU: European Union; FU: follow-up; GLM: generalized linear model; GSVM: Support Vector Machine with Gaussian Kernel; HF: heart failure; HFpEF: heart failure with preserved ejection fraction; HFrEF: heart failure due to reduced ejection fraction; LV: left ventricular; MCC: Matthews correlation coefficient; NYHA: New York Heart Association; PCC: Pearson correlation coefficient; PR: precision-recall; RF: Random Forests; ROC: receiver operating characteristic; SC: serum creatinine; SNPs: single-nucleotide polymorphisms; SVM: Support Vector Machine; TN rate: true negative rate; TP rate: true positive rate; $X G B$ : eXtreme Gradient Boosting

\section{Acknowledgments}

The authors also thank Homaira Hamidzada (University of Toronto) and Jamie Anderson for their help in the English proof-reading of this manuscript, and Tim Tripp (Library and Information Services at University Health Network) for his support.

\section{Authors' contributions}

DC retrieved the dataset, conceived the study, performed the biostatistics analysis, compared the results of the biostatistics feature rankings and the machine learning feature rankings, wrote the corresponding sections of the manuscript, and revised the final version of the manuscript. GJ performed the machine learning feature ranking and the machine learning classification based on the top two features, did the literature review, wrote the corresponding sections of the manuscript, and revised the final version of the manuscript. Both the authors read and approved the final manuscript version.

\section{Funding}

This study was supported by an article processing charge waiver generously provided by the Springer Nature Waivers Team. The Springer Nature Waivers Team had no role in the design of the study and collection, analysis, and interpretation of data and in writing the manuscript.

\section{Availability of data and materials}

The dataset used in this project [66] is publicly available under the Creative Commons Attribution 4.0 International (CC BY 4.0) license at: https://plos. figshare.com/articles/Survival_analysis_of_heart_failure_patients_A_case_ study/5227684/1
Our software code is publicly available under the GNU General Public License v3.0 at: https://github.com/davidechicco/cardiovascular_heart_disease

\section{Ethics approval and consent to participate}

The original study containing the dataset analyzed in this manuscript was approved by the Institutional Review Board of Government College University (Faisalabad, Pakistan), and states that the principles of Helsinki Declaration were followed [52].

\section{Consent for publication \\ Not applicable.}

\section{Competing interests}

The authors declare they have no competing interests.

\section{Author details}

${ }^{1}$ Krembil Research Institute, Toronto, Ontario, Canada. ${ }^{2}$ Fondazione Bruno Kessler, Trento, Italy.

Received: 12 August 2019 Accepted: 14 January 2020

Published online: 03 February 2020

References

1. World Health Organization, World Heart Day. https://www.who.int/ cardiovascular_diseases/world-heart-day/en/. Accessed 7 May 2019.

2. The Guardian. UK heart disease fatalities on the rise for first time in 50 years. https://www.theguardian.com/society/2019/may/13/heartcirculatory-disease-fatalities-on-rise-in-uk. Accessed 25 Oct 2019.

3. National Heart Lung and Blood Institute (NHLBI). Heart failure. https:// www.nhlbi.nih.gov/health-topics/heart-failure. Accessed 20 June 2019.

4. Meng F, Zhang Z, Hou X, Qian Z, Wang Y, Chen Y, Wang Y, Zhou Y, Chen Z, Zhang $X$, Yang J, Zhang J, Guo J, Li K, Chen L, Zhuang R, Jiang H, Zhou W, Tang S, Wei Y, Zou J. Machine learning for prediction of sudden cardiac death in heart failure patients with low left ventricular ejection fraction: study protocol for a retroprospective multicentre registry in China. Br Med J (BMJ) Open. 2019;9(5):023724.

5. Nauta JF, Jin X, Hummel YM, Voors AA. Markers of left ventricular systolic dysfunction when left ventricular ejection fraction is normal. Eur J Heart Fail. 2018;20:1636-8.

6. Pfeffer MA, Braunwald E. Treatment of heart failure with preserved ejection fraction. reflections on its treatment with an aldosterone antagonist. J Am Med Assoc (JAMA) Cardiol. 2016;1(1):7-8.

7. Mesquita ET, Grion DC, Kubrusly MC, Silva BBFF, Santos ÉAR. Phenotype mapping of heart failure with preserved ejection fraction. Int J Cardiovasc Sci. 2018;31(6):652-61.

8. Nanayakkara S, Kaye DM. Targets for heart failure with preserved ejection fraction. Clin Pharmacol Ther. 2017;102:228-37.

9. Katz DH, Deo RC, Aguilar FG, Selvaraj S, Martinez EE, Beussink-Nelson L, Kim K-YA, Peng J, Irvin MR, Tiwari H, Rao DC, Arnett DK, Shah SJ. Phenomapping for the identification of hypertensive patients with the myocardial substrate for heart failure with preserved ejection fraction. J Cardiovasc Transl Res. 2017;10(3):275-84.

10. Lewis GA, Schelbert EB, Williams SG, Cunnington C, Ahmed F, McDonagh TA, Miller CA. Biological phenotypes of heart failure with preserved ejection fraction. J Am Coll Cardiol. 2017;70(17):2186-200.

11. Raphael C, Briscoe C, Justin Davies ZIW, Manisty C, Sutton R, Mayet J, Francis DP. Limitations of the New York Heart Association functional classification system and self-reported walking distances in chronic heart failure. Heart. 2007:93(4):476-82.

12. Buchan TA, Ross HJ, McDonald M, Billia F, Delgado D, Duero Posada JG, Luk A, Guyatt GH, Alba AC. Physician prediction versus model predicted prognosis in ambulatory patients with heart failure. J Heart Lung Transplant. 2019;38(4):381.

13. Chapman B, DeVore AD, Mentz RJ, Metra M. Clinical profiles in acute heart failure: an urgent need for a new approach. Eur Soc Cardiol (ESC) Heart Fail. 2019:6(3):464-74.

14. Poffo MR, Assis AVd, Fracasso M, Londero Filho OM, Alves SMdM, Bald AP, Schmitt CB, Alves Filho NR. Profile of patients hospitalized for heart failure in tertiary care hospital. Int J Cardiovasc Sci. 2017;30:189-98.

15. Pandey AC, Topol EJ. Dispense with supplements for improving heart outcomes. Ann Intern Med. 2019;171:216-7. 
16. Khan $\mathrm{SU}$, Khan $\mathrm{MU}$, Riaz H, Valavoor S, Zhao D, Vaughan L, Okunrintemi V, RiazIB, Khan MS, Kaluski E, Murad MH, Blaha MJ, Guallar E, Michos ED. Effects of nutritional supplements and dietary interventions on cardiovascular outcomes: an umbrella review and evidence map. Ann Intern Med. 2019;171:190-8.

17. Chiodo L, Casula M, Tragni E, Baragetti A, Norata D, Catapano AL, on behalf of PLIC group. Profilo cardiometabolico in una coorte lombarda: lo studio PLIC. Cardio-metabolic profile in a cohort from Lombardy region: the PLIC study. Giornale Italiano di Farmacoeconomia e Farmacoutilizzazione. 2017;9(2):35-53.

18. Al'Aref SJ, Anchouche K, Singh G, Slomka PJ, Kolli KK, Kumar A, Pandey M, Maliakal G, van Rosendael AR, Beecy AN, Berman DS, Leipsic J, Nieman K, Andreini D, Pontone G, Schoepf UJ, Shaw LJ, Chang H-J, Narula J, Bax JJ, Guan Y, Min JK. Clinical applications of machine learning in cardiovascular disease and its relevance to cardiac imaging. Eur Heart J. 2018;40(24):1975-86.

19. Al'Aref SJ, Singh G, van Rosendael AR, Kolli KK, Ma X, Maliakal G, Pandey M, Lee BC, Wang J, Xu Z, Zhang Y, Min JK, Wong SC, Minutello RM. Determinants of in-hospital mortality after percutaneous coronary intervention: a machine learning approach. J Am Heart Assoc. 2019;8(5):011160.

20. Dunn WB, Broadhurst DI, Deepak SM, Buch MH, McDowell G, Spasic I, Ellis DI, Brooks N, Kell DB, Neysesc L. Serum metabolomics reveals many novel metabolic markers of heart failure, including pseudouridine and 2-oxoglutarate. Metabolomics. 2007;3(4):413-26.

21. Gallagher J, McCormack D, Zhou S, Ryan F, Watson C, McDonald K, Ledwidge MT. A systematic review of clinical prediction rules for the diagnosis of chronic heart failure. Eur Soc Cardiol (ESC) Heart Fail. 2019;6(3):499-508.

22. Ambale-Venkatesh $B$, Yang X, Wu CO, Liu K, Hundley GW, McClelland R, Gomes AS, Folsom AR, Shea S, Guallar E, Bluemke DA, Lima JAC. Cardiovascular event prediction by machine learning: the multi-ethnic study of atherosclerosis. Circ Res. 2017;121(9):1092-101.

23. Weng SF, Reps J, Kai J, Garibaldi JM, Qureshi N. Can machine-learning improve cardiovascular risk prediction using routine clinical data? PLoS ONE. 2017;12(4):0174944.

24. Shilaskar S, Ghatol A. Feature selection for medical diagnosis: evaluation for cardiovascular diseases. Expert Syst Appl. 2013;40(10):4146-53.

25. Panahiazar M, Taslimitehrani V, Pereira N, Pathak J. Using EHRs and machine learning for heart failure survival analysis. Stud Health Technol Informat. 2015;216:40.

26. Tripoliti EE, Papadopoulos TG, Karanasiou GS, Naka KK, Fotiadis DI. Heart failure: diagnosis, severity estimation and prediction of adverse events through machine learning techniques. Comput Struct Biotechnol J. 2017;15:26-47.

27. Ahmad T, Lund LH, Rao P, Ghosh R, Warier P, Vaccaro B, Dahlström U, O'Connor CM, Felker GM, Desai NR. Machine learning methods improve prognostication, identify clinically distinct phenotypes, and detect heterogeneity in response to therapy in a large cohort of heart failure patients. J Am Heart Assoc. 2018;7(8):008081.

28. Samad MD, Ulloa A, Wehner GJ, Jing L, Hartzel D, Good CW, Williams BA, Haggerty CM, Fornwalt BK. J Am Coll Cardiol (JACC) CardiovasC Interv. 2019;12:2641.

29. Sengupta PP, Kulkarni H, Narula J. Prediction of abnormal myocardial relaxation from signal processed surface ECG. J Am Coll Cardiol. 2018;71(15):1650-60

30. Krittanawong C, Johnson KW, Rosenson RS, Wang Z, Aydar M, Baber U, Min JK, Tang WHW, Halperin JL, Narayan SM. Deep learning for cardiovascular medicine: a practical primer. Eur Heart J. 2019;40:2058-73.

31. Poolsawad N, Moore L, Kambhampati C, Cleland JGF. Issues in the mining of heart failure datasets. Int J Autom Comput. 2015;11 (2):162-79.

32. Buzaev IV, Plechev W, Nikolaeva IE, Galimova RM. Artificial intelligence: neural network model as the multidisciplinary team member in clinical decision support to avoid medical mistakes. Chron Dis Transl Med. 2016;2(3):166-72.

33. Benjamins J-W, Hendriks T, Knuuti J, Juarez-Orozco LE, van der Harst P. A primer in artificial intelligence in cardiovascular medicine. Neth Heart J. 2019;27:392-402.

34. Bello GA, Dawes TJW, Duan J, Biffi C, de Marvao A, Howard LSGE, Gibbs JSR, Wilkins MR, Cook SA, Rueckert D, O'Regan DP.
Deep-learning cardiac motion analysis for human survival prediction. Nat Mach Intell. 2019;1(2):95-104

35. Smith DH, Johnson ES, Thorp ML, Yang X, Petrik A, Platt RW, Crispell K. Predicting poor outcomes in heart failure. Permanente J. 2011;15(4):4-11.

36. Dokainish H, Teo K, Zhu J, Roy A, AlHabib KF, ElSayed A, Palileo-Villaneuva L, Lopez-Jaramillo P, Karaye K, Yusoff K, Orlandini A, Sliwa K, Mondo C, Lanas F, Prabhakaran D, Badr A, Elmaghawry M, Damasceno A, Tibazarwa K, Belley-Cote E, Balasubramanian K, Islam S, Yacoub MH, Huffman MD, Harkness K, Grinvalds A, McKelvie R, Bangdiwala SI, Yusuf S, Campos R, Chacón C, Cursack G, Diez F, Escobar C, Garcia C, Vilamajo OG, Hominal M, Ingaramo A, Kucharczuk G, Pelliza M, Rojas A, Villani A, Zapata G, Bourke P, Lanas F, Nahuelpan $L$, Olivares C, Riquelme R, Ai F, Bai X, Chen X, Chen Y, Gao M, Ge C, He $Y$, Huang $W$, Jiang $H$, Liang $T$, Liang $X$, Liao $Y$, Liu S, Luo $Y$, Lu L, Qin S, Tan G, Tan H, Wang T, Wang X, Wei F, Xiao F, Zhang B, Zheng T, Mendoza JLA, Anaya MB, Gomez E, de Salazar DIM, Quiroz F, Rodríguez MJ, Sotomayor MS, Navas AT, León MB, Montalvo LAF, Jaramillo ML, Patiño EP, Perugachi C, Trujillo Cruz F, Elmaghawry M, Wagdy K, Bhardwaj AK, Chaturvedi V, Gokhale GK, Gupta R, Honnutagi R, Joshi P, Ladhani S, Negi PC, Roy A, Reddy N, Abdullah A, Hassan MRA, Balasinga M, Kasim S, Tan WY, Yusoff K, Damasceno A, Banze R, Calua E, Novela C, Chemane J, Akintunde AA, Ansa V, Gbadamosi H, Karaye KM, Mbakwem A, Mohammed S, Nwafor E, Ojji D, Olunuga T, Sa'idu BOH, Umuerri E, Alcaraz J, Palileo-Villanueva L, Palomares E, Timonera MR, Badr A, Alghamdi S, Alhabib K, Almasood A, Alsaif S, Elasfar A, Ghabashi A, Mimish L, Bester F, Kelbe D, Klug E, Sliwa K, Tibarzawa K, Abdalla OE, Dimitri ME, Mustafa H, Osman O, Saad A, Mondo C. Global mortality variations in patients with heart failure: results from the International Congestive Heart Failure (INTER-CHF) prospective cohort study. Lancet Glob Health. 2017;5:665-72.

37. Voors AA, OuwerkerkW, Zannad F, van Veldhuisen DJ, Samani NJ, Ponikowski P, Ng LL, Metra M, ter Maaten JM, Lang CC, Hillege HL, van der Harst P, Filippatos G, Dickstein K, Cleland JG, Anker SD, Zwinderman AH. Development and validation of multivariable models to predict mortality and hospitalization in patients with heart failure. Eur J Heart Fail. 2017;19:627-34.

38. Levy WC, Mozaffarian D, Linker DT, Sutradhar SC, Anker SD, Cropp AB, Anand I, Maggioni AP, Burton P, Sullivan MD, Pitt B, Poole-wilson PA Mann DL, Packer M. The Seattle heart failure model: prediction of survival in heart failure. Circulation. 2006;113(11):1424-33.

39. Sakamoto M, Fukuda H, Kim J, Ide T, Kinugawa S, Fukushima A, Tsutsui H, Ishii A, Ito S, Asanuma H, Asakura M, Washio T, Kitakaze M. The impact of creating mathematical formula to predict cardiovascular events in patients with heart failure. Sci Rep. 2018;8(1):3986.

40. Alba AC, Agoritsas T, Jankowski M, Courvoisier D, Walter SD, Guyatt $\mathrm{GH}$, Ross HJ. Risk prediction models for mortality in ambulatory patients with heart failure: a systematic review. Circ Heart Fail. 2013;6:881-89.

41. Yap J, Chia SY, Lim FY, Allen JC, Teo L, Sim D, Go YY, Jaufeerally FR, Seow M, Kwok B, Liew R, Lam CS, Ching CK. The Singapore heart failure risk score: prediction of survival in Southeast Asian patients. Ann Acad Med Singap. 2019;48:86-94.

42. Yap J, Lim FY, Chia SY, Allen Jr. JC, Jaufeerally FR, Macdonald MR, Chai P, Loh SY, Lim P, Zaw MWW, Teo L, Sim D, Lam CSP. Prediction of survival in Asian patients hospitalized with heart failure: validation of the OPTIMIZE-HF risk score. J Card Fail. 2019;25(7):571-5.

43. Kasahara S, Sakata Y, Sakata Y, Nochioka K, Tay WT, Claggett BL, Abe R, Oikawa T, Sato M, Aoyanagi H, Miura M, Shiroto T, Takahashi J, Sugimura K, Teng T-HK, Miyata S, Shimokawa H. The 3A3B score: the simple risk score for heart failure with preserved ejection fraction - A report from the CHART-2 Study. Int J Cardiol. 2019;284:42-9.

44. Miyagawa S, Pak K, Hikoso S, Ohtani T, Amiya E, Sakata Y, Ueda S, Takeuchi M, Komuro I, Sawa Y. Japan heart failure model - Derivation and accuracy of survival prediction in Japanese heart failure patients. Circ Rep. 2019;1(1):29-34.

45. Boralkar KA, Kobayashi Y, Moneghetti K, Pargaonkar VS, Tuzovic M, Krishnan G, Wheeler MT, Banerjee D, Kuznetsova T, Horne BD, Knowlton KU, Heidenreich PA, Haddad F. Improving risk stratification in heart failure with preserved ejection fraction by combining two validated risk scores. Open Heart. 2019;6(1):e000961.

46. Kouwert IJM, Bakker EA, Cramer MJ, Snoek JA, Eijsvogels TMH. Comparison of MAGGIC and MECKI risk scores to predict mortality after 
cardiac rehabilitation among Dutch heart failure patients. Eur J Prev Cardiol. 2019;First published online:26.

47. Canepa M, Fonseca C, Chioncel O, Laroche C, Crespo-Leiro MG, Coats AJS, Mebazaa A, Piepoli MF, Tavazzi L, Maggioni AP, Crespo-Leiro M, Anker S, Mebazaa A, Coats A, Filippatos G, Ferrari R, Maggioni AP, Piepoli MF, Amir O, Chioncel O, Dahlström U, Delgado Jimenez JF, Drozdz J, et al. Performance of prognostic risk scores in chronic heart failure patients enrolled in the European society of cardiology heart failure long-term registry. J Am Coll Cardiol (JACC) Heart Fail. 2018;6(6): 452-62.

48. Straw S, Byrom R, Gierula J, Paton MF, Koshy A, Cubbon R, Drozd M, Kearney M, Witte KK. Predicting one-year mortality in heart failure using the 'surprise question': a prospective pilot study. Eur J Heart Fail. 2019;21(2):227-34

49. Dauriz M, Mantovani A, Bonapace S, Verlato G, Zoppini G, Bonora E, Targher G. Prognostic impact of diabetes on long-term survival outcomes in patients with heart failure: a meta-analysis. Diabetes Care. 2017:40(11):1597-605.

50. Segar MW, Vaduganathan M, Patel KV, McGuire DK, Butler J, Fonarow GC, Basit M, Kannan V, Grodin JL, Everett B, Willett D, Berry J, Pandey A. Machine learning to predict the risk of incident heart failure hospitalization among patients with diabetes: the WATCH-DM risk score. Diabetes Care. 2019;42(12):2298-306.

51. Son MK, Lim N-K, Park H-Y. Predicting stroke and death in patients with heart failure using CHA2DS2-VASC score in Asia. BMC Cardiovasc Disord. 2019;19(1):193.

52. Ahmad T, Munir A, Bhatti SH, Aftab M, Raza MA. Survival analysis of heart failure patients: a case study. PLoS ONE. 2017;12(7):0181001.

53. Fitrianto A, Jiin RLT. Several types of residuals in Cox regression model: an empirical study. Int J Math Anal. 2013;7:2645-54.

54. Kleinbaum DG, Klein M. Kaplan-Meier survival curves and the log-rank test. Heidelberg, Germany: Springer; 2012, pp. 55-96.

55. Wilkinson M, Dumontier M, Aalbersberg I, Appleton G, Axton M, Baak A, Blomberg N, Boiten J, da Silva Santos L, Bourne P, Bouwman J, Brookes A, Clark T, Crosas M, Dillo I, Dumon O, Edmunds S, Evelo C, Finkers R, Gonzalez-Beltran A, Gray A, Groth P, Goble C, Grethe J, Heringa J, 't Hoen P, Hooft R, Kuhn T, Kok R, Kok J, Lusher S, Martone M, Mons A, Packer A, Persson B, Rocca-Serra P, Roos M, van Schaik R, Sansone S, Schultes E, Sengstag T, Slater T, Strawn G, Swertz M, Thompson M, van Der Lei J, van Mulligen E, Velterop J, Waagmeester A, Wittenburg P, Wolstencroft K, Zhao J, Mons B. The FAIR Guiding Principles for scientific data management and stewardship. Sci Data. 2016;3:160018.

56. Zahid FM, Ramzan S, Faisal S, Hussain I. Gender based survival prediction models for heart failure patients: a case study in Pakistan. PLOS ONE. 2019;14(2):0210602.

57. Núñez J, Garcia S, Núñez E, Bonanad C, Bodí V, Miñana G, Santas E, Escribano D, Bayes-Genis A, Pascual-Figal D, Chorro FJ, Sanchis J. Early serum creatinine changes and outcomes in patients admitted for acute heart failure: the cardio-renal syndrome revisited. Eur Heart J Acute Cardiovasc Care. 2017;6(5):430-40.

58. Akhter MW, Aronson D, Bitar F, Khan S, Singh H, Singh RP, Burger AJ, Elkayam $U$. Effect of elevated admission serum creatinine and its worsening on outcome in hospitalized patients with decompensated heart failure. Am J Cardiol. 2004;94:957-60.

59. Brisco MA, Zile MR, Hanberg JS, Wilson FP, Parikh CR, Coca SG, Tang WHW, Testani JM. Relevance of changes in serum creatinine during a heart failure trial of decongestive strategies: insights from the DOSE trial. J Card Fail. 2016;22(10):753-60.

60. Vistarini N, Deschamps A, Cartier R. Preoperative creatinine clearance affects long-term survival after off-pump coronary artery bypass surgery. Can J Cardiol. 2014;30:238-9.

61. Tomaselli Muensterman E, Tisdale JE. Predictive analytics for identification of patients at risk for QT interval prolongation: a systematic review. Pharmacotherapy. 2018;38(8):813-21.

62. Kosztin AA, Tokodi M, Toser Z, Schwertner W, Boros A, Kovacs A, Perge P, Szeplaki G, Geller L, Merkely B. Utilization of machine learning to identify gender-specific patterns in short-and long-term mortality after cardiac resynchronization therapy. In: Proceedings of the Heart Failure 2019 Congress, vol. 1; 2019. p. 834.

63. Stasiak MM, Rozentryt P, Jankowska E, Retwinski A, Straburzynska-Migaj E, Nowalany-Kozielska E, Ponikowski P, Mirek-Bryniarska E, Polonski L,
Drozdz J. Renal failure in patients with heart failure - analysis based on ESC-HF Pilot survey. Eur Heart J. 2013;34(Suppl 1):645.

64. Sutherland SM, Chawla LS, Kane-Gill S, Hsu RK, Kramer AA, Goldstein SA, Kellum JA, Ronco C, Bagshaw SM, the 15 ADQI Consensus Group. Utilizing electronic health records to predict acute kidney injury risk and outcomes: workgroup statements from the 15th ADQI Consensus Conference. Can J Kidney Health Dis. 2016;3:11.

65. Lee H-C, Yoon H-K, Nam K, Cho YJ, Kim TK, Kim WH, Bahk J-H. Derivation and validation of machine learning approaches to predict acute kidney injury after cardiac surgery. J Clin Med. 2018;7:322.

66. Ahmad T, Munir A, Bhatti SH, Aftab M, Ali Raza M. Survival analysis of heart failure patients: a case study. Dataset. https://plos.figshare.com/ articles/Survival_analysis_of_heart_failure_patients_A_case_study/ 5227684/1. Accessed 25 Jan 2019.

67. Bredy C, Ministeri M, Kempny A, Alonso-Gonzalez R, Swan L, Uebing A, Diller G-P, Gatzoulis MA, Dimopoulos K. New York Heart Association (NYHA) classification in adults with congenital heart disease: relation to objective measures of exercise and outcome. Eur Heart J - Qual Care Clin Outcomes. 2017:4(1):51-8.

68. Johns Hopkins Rheumatology. Creatine Phosphokinase (CPK). https:// www.hopkinslupus.org/lupus-tests/clinical-tests/creatinephosphokinase-cpk/. Accessed 25 Jan 2019.

69. Stephens C. What is a creatinine blood test? https://www.healthline com/health/creatinine-blood. Accessed 25 Jan 2019.

70. Case-Lo C. What is a sodium blood test? https://www.healthline.com/ health/sodium-blood. Accessed 25 Jan 2019.

71. Seber GA, Lee AJ. Linear Regression Analysis, Wiley Series in Probability and Statistics. vol. 329. Hoboken: John Wiley and Sons; 2012.

72. Breiman L. Random forests. Mach Learn. 2001;45:5-32.

73. Holte RC. Very simple classification rules perform well on most commonly used datasets. Mach Learn. 1993;11 (1):63-90.

74. Loh W-Y. Classification and regression trees. Wiley Interdiscip Rev Data Min Knowl Disc. 2011;1(1):14-23.

75. LeCun Y, Bengio Y, Hinton G. Deep learning. Nature. 2015;521(7553):436

76. Amari S-I, Wu S. Improving support vector machine classifiers by modifying kernel functions. Neural Netw. 1999;12(6):783-9.

77. Cover TM, Hart PE. Nearest neighbor pattern classification. IEEE Trans Inf Theory. 1967:13(1):21-7.

78. Rish I. An empirical study of the naive Bayes classifier. In: Proceedings of IJCAI 2001 - the 17th International Joint Conferences on Artificial Intelligence Workshop on Empirical Methods in Artificial Intelligence, vol. 3. Menlo Park: American Association for Artificial Intelligence; 2001. p. $41-46$.

79. Chen T, Guestrin C. XgBoost: a scalable tree boosting system. In: Proceedings of KDD 2016 - the 22nd ACM SIGKDD International Conference on Knowledge Discovery and Data Mining. New York City: Association for Computing Machinery (ACM); 2016. p. 785-794.

80. Matthews BW. Comparison of the predicted and observed secondary structure of T4 phage lysozyme. Biochim Biophys Acta (BBA) - Protein Struct. 1975;405(2):442-51

81. Saito T, Rehmsmeier M. The precision-recall plot is more informative than the ROC plot when evaluating binary classifiers on imbalanced datasets. PLoS ONE. 2015;10(3):0118432.

82. Jurman G, Riccadonna S, Furlanello C. A comparison of MCC and CEN error measures in multi-class prediction. PLoS ONE. 2012;7(8):41882.

83. Chicco D. Ten quick tips for machine learning in computational biology. BioData Min. 2017;10(35):1-17.

84. Chicco D, Jurman $\mathrm{G}$. The advantages of the Matthews correlation coefficient (MCC) over F1 score and accuracy in binary classification evaluation. BMC Genomics. 2020;21(1):6.

85. Wilcoxon F. Individual comparisons by ranking methods. Biom Bull. 1945; 1(6):80-3.

86. Benesty J, Chen J, Huang Y, Cohen I. Pearson correlation coefficient. In: Noise Reduction in Speech Processing. Heidelberg: Springer; 2009. p. 1-4.

87. McHugh ML. The chi-square test of independence. Biochemia Medica. 2013;23(2):143-9.

88. Shapiro SS, Wilk MB. An analysis of variance test for normality (complete samples). Biometrika. 1965;52(3/4):591-611.

89. Light C. Tutorial: Pearson's Chi-square test for independence. https:// www.ling.upenn.edu/ clight/chisquared.htm. Accessed 7 May 2019. 
90. Patrício M, Pereira J, Crisóstomo J, Matafome P, Gomes M, Seiça R, Caramelo F. Using resistin, glucose, age and BMI to predict the presence of breast cancer. BMC Cancer. 2018;18(1):29.

91. Breiman L, Cutler A. Random forests - Gini importance. https://www stat.berkeley.edu/ breiman/RandomForests/cc_home.htm\#giniimp. Accessed 10 Apr 2019.

92. Chicco D, Rovelli C. Computational prediction of diagnosis and feature selection on mesothelioma patient health records. PLOS ONE. 2019;14(1):0208737.

93. Kononenko I. Estimating attributes: analysis and extensions of RELIEF. In: Proceedings of ECML 1994 - the 7th European Conference on Machine Learning. Heidelberg: Springer; 1994. p. 171-82.

94. Robnik-Sikonja M, Kononenko I. An adaptation of Relief for attribute estimation in regression. In: Proceedings of ICML 1997 - the 14th International Conference on Machine Learning. Burlington: Morgan Kaufmann Publishers; 1997. p. 296-304.

95. Urbanowicz RJ, Olson RS, Schmitt P, Meeker M, Moore JR. Benchmarking relief-based feature selection methods for bioinformatics data mining. J Biomed Inform. 2018;85:168-88.

96. Brown LE, Tsamardinos I, Aliferis CF. A novel algorithm for scalable and accurate Bayesian network learning. In: Proceedings of MEDINFO 2004 the 11th World Congress on Medical Informatics. Amsterdam: IOS Press; 2004. p. 711-5.

97. Lagani V, Athineou G, Farcomeni A, Tsagris M, Tsamardinos I. Feature selection with the R package MXM: discovering statistically equivalent feature subsets. J Stat Softw Artic. 2017;80(7):1-25.

98. Borboudakis G, Tsamardinos I. Forward-backward selection with early dropping. J Mach Learn Res. 2019;20(1):276-314.

99. Breiman L, Friedman JH, Ohlsen RA, Stone CJ. Classification and Regression Trees. The Wadsworth Statistics Probability Series. Boston: Wadsworth Publishing; 1984, p. 358.

100. Cortes C, Vapnik VN. Support-vector networks. Mach Learn. 1995;20(3): 273-97.

101. Friedman JH, Hastie T, Tibshirani R. Additive logistic regression: a statistical view of boosting (with discussion and a rejoinder by the authors). Ann Stat. 2000;28(2):337-407.

102. Friedman JH. Greedy function approximation: a gradient boosting machine. Ann Stat. 2001;29(5):1189-232.

103. de Borda J-C. Mémoire sur les élections au scrutin. Histoire de I'Académie Royale des Sciences. 1784;Jg. 1781:657-65.

104. Barla A, Galea A, Furlanello C, Jurman G, Paoli S, Merler S. Algebraic stability indicators for ranked lists in molecular profiling. Bioinformatics. 2007;24(2):258-64

105. Guyon I, Weston J, Barnhill S, Vapnik V. Gene selection for cancer classification using support vector machines. Mach Learn. 2002;46(1-3): 389-422.

106. Liu S, Zheng H, Feng Y, Li W. Prostate cancer diagnosis using deep learning with 3D multiparametric MRI. In: Proceedings of Medical Imaging 2017: Computer-Aided Diagnosis, vol. 10134. Bellingham: International Society for Optics and Photonics (SPIE); 2017. p. 1013428.

107. Mehta CR, Patel NR. Exact logistic regression: theory and examples. Stat Med. 1995;14(19):2143-60.

108. Liaw A, Wiener M. Classification and regression by randomForest. $R$ News. 2002;2(3):18-22.

109. Chicco D, Ciceri E, Masseroli M. Extended Spearman and Kendall coefficients for gene annotation list correlation. In: Proceedings of the International Meeting on Computational Intelligence Methods for Bioinformatics and Biostatistics (CIBB 2014). Springer; 2014. p. 19-32.

110. Sculley D. Rank aggregation for similar items. In: Proceedings of the 2007 SIAM International Conference on Data Mining. Philadelphia: Society for Industrial and Applied Mathematics; 2007. p. 587-592.

111. Yunus I, Fasih A, Wang Y. The use of procalcitonin in the determination of severity of sepsis, patient outcomes and infection characteristics. PLOS ONE. 2018;13(11):0206527.

112. Masino AJ, Harris MC, Forsyth $D$, Ostapenko $S$, Srinivasan L, Bonafide CP, Balamuth F, Schmatz M, Grundmeier RW. Machine learning models for early sepsis recognition in the neonatal intensive care unit using readily available electronic health record data. PLoS ONE. 2019;14(2): 0212665.

113. Aushev A, Ripoll VR, Vellido A, Aletti F, Pinto BB, Herpain A, Post EH, Medina ER, Ferrer R, Baselli G. Feature selection for the accurate prediction of septic and cardiogenic shock ICU mortality in the acute phase. PLoS ONE. 2018;13(11):0199089.

114. Fernandes K, Chicco D, Cardoso JS, Fernandes J. Supervised deep learning embeddings for the prediction of cervical cancer diagnosis. PeerJ Comput Sci. 2018;4:154.

115. Maggio V, Chierici M, Jurman G, Furlanello C. Distillation of the clinical algorithm improves prognosis by multi-task deep learning in high-risk neuroblastoma. PLoS ONE. 2018;13(12):0208924.

116. Kueffner R, Zach N, Bronfeld M, Norel R, Atassi N, Balagurusamy V, Camillo BD, Chio A, Cudkowicz M, Dillenberger D, Garcia-Garcia J, Hardiman O, Hoff B, Knight J, Leitner ML, Li G, Mangravite L, Norman T, Wang L, the ALS Stratification Consortium, Xiao J, Fang W-C, Peng J, Yang C, Chang H-J, Stolovitzky G. Stratification of amyotrophic lateral sclerosis patients: a crowdsourcing approach. Sci Rep. 2019;9(1):690.

\section{Publisher's Note}

Springer Nature remains neutral with regard to jurisdictional claims in published maps and institutional affiliations.

\section{Ready to submit your research? Choose BMC and benefit from}

- fast, convenient online submission

- thorough peer review by experienced researchers in your field

- rapid publication on acceptance

- support for research data, including large and complex data types

- gold Open Access which fosters wider collaboration and increased citations

- maximum visibility for your research: over 100M website views per year

At $\mathrm{BMC}$, research is always in progress.

Learn more biomedcentral.com/submissions 